\title{
An Efficient Cooperation Protocol to Extend Coverage Area in Cellular Networks
}

\author{
Ahmed K. Sadek, Zhu Han, and K. J. Ray Liu \\ Department of Electrical and Computer Engineering, \\ and Institute for Systems Research \\ University of Maryland, College Park, MD 20742, USA. \\ \{aksadek, hanzhu, kjrliu\}@eng.umd.edu
}

\begin{abstract}
In this paper, we propose a bandwidth efficient cooperation protocol to extend coverage area in cellular networks. An important question, however, in implementing any cooperation protocol is how to assign cooperating partners. We introduce a BS-controlled relay-assignment algorithm to assign cooperating users. First, we derive a lower bound on the outage performance of any practical relay assignment protocol. The lower bound can be achieved by a hypothetical Genie-aided protocol that places a relay at the optimal theoretical location for every source in the network. Then we describe and analyze the performance of the base-station/access-point (BS/AP) controlled protocol. The BS/AP-controlled protocol can be implemented at the BS or the AP, and it tries to emulate the Genie-Aided protocol. Simulation results for rural cellular systems are provided. By utilizing the proposed protocol, simulation results indicate a significant gain in coverage area over the direct transmission scheme under fairly the same bandwidth efficiency and fixed average transmitted power. For the rural cellular system case, coverage extension of about $250 \%$ can be achieved by the BS/AP-controlled protocol.
\end{abstract}

\section{INTRODUCTION}

The growing demands of wireless applications require the service providers to provide reliable communications anytime anywhere. This poses challenges to the deployment of cellular networks especially in rural areas. One solution to this problem is to increase the number of basestaions, however, this is not a cost effective solution. Another solution is to increase the transmitted power from the wireless terminals. This is not also a preferable solution as the transmitted power of the wireless terminals is usually limited, besides increasing the transmitted power introduces health hazards.

The cooperative communication concept has been recently proposed [1], [2], [3], [4], [5], [6], [7], introducing a new communication paradigm to the research community. The basic idea is to explore the broadcast nature of the wireless channel. In almost all of the previous works, however, the cooperating relays are just assumed to exist and are already coupled with the source nodes in the network. These works also assumed fixed channel variances between all of the nodes in the network, which implies a fixed network topology. If the random

This work was supported in part by CTA-ARL DAAD 190120011 and by MURI AFOSR F496200210217. users' spatial distribution, and the associated propagation path losses between different nodes in the network are taken into consideration, then these assumptions, in general, are no longer valid.

In this paper, we address the relay-assignment problem and propose a practical relay-assignment algorithm for implementing cooperative diversity protocols to extend coverage area in wireless networks. To better assess the performance of the proposed protocol, we first derive a lower bound on the outage probability of any practical relay-assignment protocol. The lower bound is derived by assuming a Genie-aided protocol in which a Genie places a relay at the optimal theoretical location, which we show to be at the mid-point of the source-destination link, for each source in the network. Next, we describe the BS/AP-controlled protocol to implement the relay-assignment function that tries to emulate the performance of the GenieAided protocol. By finding the node that is nearest to the midpoint of each source-destination pair, the BS/AP-controlled protocol assigns this node as a relay to this source. An upper bound on the outage probability of the BS/AP-controlled protocol is derived and is shown to be close to the Genie-Aided protocol performance via simulations. Once the relay is assigned, any cooperation scheme can be employed. In this paper, we consider a modified version of the incremental relaying protocol proposed in [1]. As will be demonstrated later, the loss in the bandwidth efficiency is negligible in incremental relaying compared to that of direct transmission for practical ranges of the signal to noise ratio [1].

Furthermore, simulations are carried out to validate the theoretical results derived for the described protocols. We consider the application of the proposed protocol in coverage area extension in cellular networks. Coverage extension up to $250 \%$ can be achieved in the cellular network via the BS/AP-controlled protocol.

The rest of the paper is organized as follows. In Section II, we present the system model and describe the proposed relayassignment algorithm and cooperation protocol. In Section III, we provide outage performance analysis for all the proposed protocols. Simulation results are conducted in Section IV, and finally conclusions are drawn in Section V. 


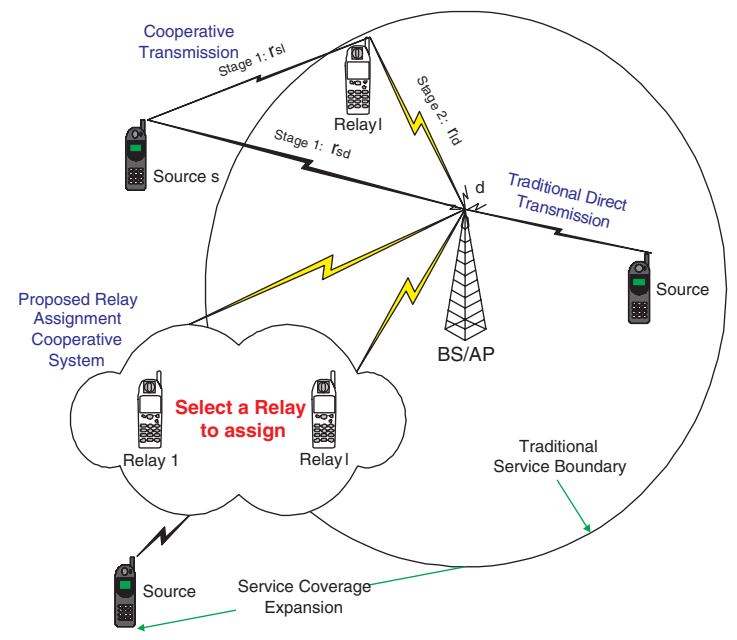

Fig. 1. Illustrating the difference between the direct and cooperative transmission schemes, and the coverage extension prospected by cooperative transmission.

\section{System Model AND RELAY-ASSIGNMENT ALGORITHMS}

We consider a wireless network, that can be a cellular system or a WLAN, with a circular cell of radius $\rho$. The BS/AP is located at the center of the cell, and $N$ users are uniformly distributed within the cell. The probability density function of the user's distance $r$ from the BS/AP is thus given by

$$
q(r)=\frac{2 r}{\rho^{2}}, \quad 0 \leq r \leq \rho,
$$

and the user's angle is uniformly distributed between $[0,2 \pi)$. The wireless link between any two nodes in the network is subject to narrowband Rayleigh fading, propagation path-loss, and additive white Gaussian noise (AWGN). The channel fades for different links are assumed to be statistically mutually independent. This is a reasonable assumption as the nodes are usually spatially well separated. For medium access, the nodes are assumed to transmit over orthogonal channels, thus no mutual interference is considered in the signal model. All nodes in the network are assumed to be equipped with single-element antennas, and transmission at all nodes is constrained to the halfduplex mode, i.e., any terminal cannot transmit and receive simultaneously [1].

In the direct transmission scheme, which is employed in current wireless networks, each user transmits his signal directly to the BS/AP as depicted in Fig. 1. The signal to noise ratio received at the destination $d$ (BS/AP) from source user $s$, can be modeled as

$$
\operatorname{SNR}\left(r_{s d}\right)=\frac{\left|h_{s d}\right|^{2} K r_{s d}^{-\eta} P_{T D}}{N_{o}} .
$$

where $P_{T D}$ is the transmitted signal power in the direct transmission mode, $h_{i j}$ is the channel fading gain between two terminals $i$ and $j-i, j$ are any two terminals in the network. The channel fade of any link is modeled throughout the paper as a zero mean circularly symmetric complex Gaussian random variable with unit variance. In (2), $K$ is a constant that depends on the antennas design, $\eta$ is the path loss exponent, and $r_{s d}$ is the distance between the two terminals. $K, \eta$, and $P_{T D}$ are assumed to be the same for all users. All the noise components throughout the paper are modeled as white Gaussian noise (AWGN) with variance $N_{o}$.

In this paper we characterize the system performance in terms of outage probability. Outage is defined as the event that the received SNR falls below a certain threshold $\gamma$, hence, the probability of outage $P_{O}$ is defined as,

$$
\mathcal{P}_{O}=\mathcal{P}(\operatorname{SNR}(r) \leq \gamma)
$$

The SNR threshold $\gamma$ is determined according to the application and the transmitter/receiver structure. If the received SNR is higher than the threshold $\gamma$, the receiver is assumed to be able to decode the received message with negligible probability of error. If an outage occurs, the packet is considered lost. The main drawback of direct transmission is that the BS/AP receives only one copy of the message from the source, which makes the communication susceptible to failure due to fading. On the other hand, when cooperative diversity is employed, the $\mathrm{BS} / \mathrm{AP}$ can receive more than one copy of the message as depicted in Fig. 1. This figure depicts a cooperation scenario where a source can employ another node (relay) in order to forward his information to the destination, which creates two-path diversity.

In this work, we adopt for the cooperating protocol a modified version of the incremental relaying protocol in [1]. In this modified protocol, if a user's packet is lost, the BS/AP broadcasts negative acknowledgement (NACK) so that the relay assigned to this user can re-transmit this packet again. The relay will only transmit the packet if it is capable of capturing the packet, i.e., if the received SNR at the relay is above the threshold. In practice, this can be implemented by utilizing a cyclic redundancy check (CRC) code in the transmitted packet. This is the first difference between the modified and original incremental relaying protocol in [1] which employs amplify-and-forward at the relay. The signal received from the source to the destination $d$ and the relay $l^{1}$ in the first stage can be modeled as,

$$
\begin{aligned}
& y_{s d}=\sqrt{P_{T C} K r_{s d}^{-\eta}} h_{s d} x+n_{s d}, \\
& y_{s l}=\sqrt{P_{T C} K r_{s l}^{-\eta}} h_{s l} x+n_{s l},
\end{aligned}
$$

where $P_{T C}$ is the transmission power in the cooperative mode and will be determined rigourously later in order to ensure the same average transmitted power in both the direct and cooperative scenarios. If the SNR of the signal received at the destination from the source falls below the threshold $\gamma$, the destination asks for a second copy from the relay. Then if the relay was able to receive the packet from the source correctly, it forwards it to the destination

\footnotetext{
${ }^{1}$ We denote the relay by $l$ not to confuse with $r$ that denotes distance.
} 


$$
y_{l d}=\sqrt{P_{T C} K r_{l d}^{-\eta}} h_{l d} x+n_{l d},
$$

A second difference between our modified protocol and the conventional incremental relaying in [1], is that in case of packet failure in the first transmission from the source, the BS/AP does not store this packet to combine it later with the packet received from the relay. This was assumed in most of the previous works, however, a crucial implication of this assumption is that the destination has to store an analog form of the signal, which is not practical. This could be practically solved, for example, by storing a quantized version of the signal, and the quantization noise should then be taken into account in the analysis. Note that the relay-assignment algorithms that we develop in this paper can be applied with any cooperation scheme.

\section{A. Relay-Assignment Protocols}

In this subsection, we propose a practical relay-assignment protocol for nodes cooperation. Moreover, we describe a Genie-Aided protocol whose performance acts as a lowerbound on the outage performance of any practical relay assignment algorithm.

- Genie-Aided Protocol: In this protocol, we first determine the optimum relay location that minimizes the outage probability for a fixed source-destination pair. As will be shown later, the optimal relay location is at the midpoint of the line joining the source and destination. Our Genie-Aided protocol works like this: For any source node in the network, a Genie is going to place a relay for this source at the optimal location. Hence, for every node in the network, there exists a relay at the optimal location that minimizes the outage probability. The performance of the Genie-Aided protocol is thus a lower bound on the outage performance of any other practical relay assignment algorithm.

- BS/AP-Controlled Protocol: The Genie-Aided protocol is a hypothetical system that cannot be implemented in a real network. However, we can get some insights from the operation of the Genie-Aided protocol to design a practical relay-assignment algorithm that can be implemented in a real network and can provide outage performance that is close to the Genie-Aided protocol. We propose the BS/AP-controlled protocol that can be implemented at the $\mathrm{BS} / \mathrm{AP}$ to perform the relay assignment algorithm. The assumption here is that the BS/AP can know the locations of all the users attached to its cell. This can be done via network-aided positioning techniques, (see [8] for a survey on localization algorithms). To assign a relay to help a certain source, the BS/AP is going to locate the node that is closet to the mid point of the line joining this source and the BS/AP. Fig. 2 demonstrates an example for this protocol where the closet node to the mid-point $c$ of the $s-d$ line is at a distance $r_{c}$ from $c$. Once the relay is assigned it continues helping the source, and the relay assignment algorithm can be updated when the network topology changes

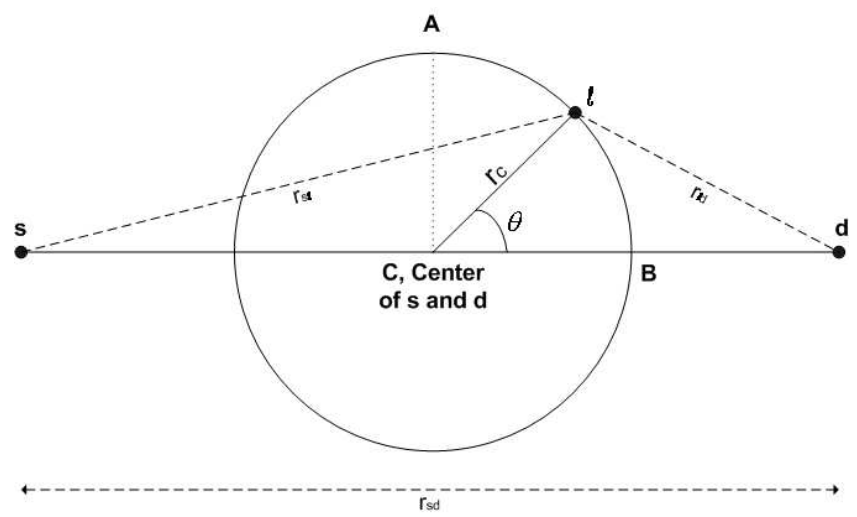

Fig. 2. Demonstrating the BS/AP-controlled protocol.

considerably. Hence, the update period is mainly determined according to the nodes' mobility model.

\section{Performance Analysis of the Proposed PROTOCOLS}

\section{A. Direct Transmission}

As discussed before, the outage is defined as the event that the received SNR is lower than a predefined threshold which we denote by $\gamma$. From the received SNR model in (2), the outage probability for the direct transmission mode $P_{O D}$ conditioned on the user's distance can be calculated as

$$
\mathcal{P}_{O D}\left(r_{s d}\right)=\mathcal{P}\left(\operatorname{SNR}\left(r_{s d}\right) \leq \gamma\right)=1-\exp \left(-\frac{N_{o} \gamma r_{s d}^{\eta}}{K P_{T D}}\right)
$$

To find the average outage probability over the cell, we need to average over the user distribution in (1). The average outage probability is thus given by

$$
\begin{aligned}
\mathcal{P}_{O D}= & \int_{0}^{\rho} \mathcal{P}_{O D}\left(r_{s d}\right) q\left(r_{s d}\right) d r_{s d} \\
& =1-\frac{2}{\eta \rho^{2}}\left(\frac{K P_{T D}}{N_{o} \gamma}\right)^{\frac{2}{\eta}} \Gamma\left(\frac{2}{\eta}, \frac{N_{o} \gamma \rho^{\eta}}{K P_{T D}}\right),
\end{aligned}
$$

where $\Gamma(\cdot, \cdot)$ is the incomplete Gamma function, and it is defined as [9],

$$
\Gamma(a, x)=\int_{0}^{x} \exp ^{-t} t^{a-1} d t
$$

\section{B. Genie-Aided Protocol}

In the Genie-Aided protocol, we consider a hypothetical system that can act as a lower bound on the outage performance of any practical relay-assignment algorithm.

Consider a source-destination pair that are $r_{s d}$ units distance apart as depicted in Fig. 1. Let us compute the conditional outage probability for given locations of the user and the helping relay. As indicated in the figure, the distances between the source and BS/AP, source and relay, and relay and BS/AP are denoted by $r_{s d}, r_{s l}$, and $r_{l d}$, respectively. Using (4), the SNR 
received at the $\mathrm{BS} / \mathrm{AP} d$ and the relay $l$ from the source $s$ is given by

$$
\begin{aligned}
& \operatorname{SNR}\left(r_{s d}\right)=\frac{\left|h_{s d}\right|^{2} K r_{s d}^{-\eta} P_{T C}}{N_{o}}, \\
& \operatorname{SNR}\left(r_{s l}\right)=\frac{\left|h_{s l}\right|^{2} K r_{s l}^{-\eta} P_{T C}}{N_{o}} .
\end{aligned}
$$

While from (5), the SNR received at the BS/AP from the relay is given by

$$
\operatorname{SNR}\left(r_{l d}\right)=\frac{\left|h_{l d}\right|^{2} K r_{l d}^{-\eta} P_{T C}}{N_{o}} .
$$

The terms $\left|h_{s d}\right|^{2},\left|h_{s l}\right|^{2}$, and $\left|h_{l d}\right|^{2}$ are mutually independent exponential random variables with unit mean. The outage probability of the cooperative transmission $P_{O C}$ conditioned on the fixed topology of the user $s$ and the relay $l$, as in Fig. 1, can be calculated as follows

$$
\begin{aligned}
& \mathcal{P}_{O C}\left(r_{s d}, r_{s l}, r_{l d}\right)=\mathcal{P}\left(\left(\operatorname{SNR}\left(r_{s d}\right) \leq \gamma\right) \cap\left(\operatorname{SNR}\left(r_{s l}\right) \leq \gamma\right)\right) \\
& +\mathcal{P}\left(\left(\operatorname{SNR}\left(r_{s d}\right) \leq \gamma\right) \cap\left(\operatorname{SNR}\left(r_{l d}\right) \leq \gamma\right) \cap\left(\operatorname{SNR}\left(r_{s l}\right)>\gamma\right)\right),
\end{aligned}
$$

where the first term corresponds to the event that both the source-BS/AP and source-relay channels are in outage, and the second term corresponds to the event that both the sourceBS/AP and relay-BS/AP channels are in outage while the source-relay channel is not. This can be shown to be given by

$$
\begin{aligned}
& \mathcal{P}_{O C}\left(r_{s d}, r_{s l}, r_{l d}\right)=\left(1-\exp \left(-\frac{N_{o} \gamma r_{s d}^{\eta}}{K P_{T C}}\right)\right)\left(1-\exp \left(-\frac{N_{o} \gamma r_{s l}^{\eta}}{K P_{T C}}\right)\right) \\
& +\left(1-\exp \left(-\frac{N_{o} \gamma r_{s d}^{\eta}}{K P_{T C}}\right)\right)\left(1-\exp \left(-\frac{N_{o} \gamma r_{l d}^{\eta}}{K P_{T C}}\right)\right) \exp \left(-\frac{N_{o} \gamma r_{s l}^{\eta}}{K P_{T C}}\right) .
\end{aligned}
$$

The above expression can be simplified as follows ${ }^{2}$

$$
\begin{array}{r}
\mathcal{P}_{O C}\left(r_{s d}, r_{s l}, r_{l d}\right)=\left(1-\exp \left(-\frac{N_{o} \gamma r_{s d}^{\eta}}{K P_{T C}}\right)\right) \\
\times\left(1-\exp \left(-\frac{N_{o} \gamma\left(r_{s l}^{\eta}+r_{l d}^{\eta}\right)}{K P_{T C}}\right)\right) .
\end{array}
$$

It can be seen that if we have the freedom to put the relay anywhere in the two-dimensional plane of the source-destination pair, then the optimal relay position should be on the line joining the source and the destination- this is because of the fact that if the relay is located at any position in the two-dimensional plane, then its distances to both the source and the destination are always larger than their corresponding projections on the straight line joining the source-destination pair. In this case, we can substitute for $r_{l d}$ by $r_{s d}-r_{s l}$. The optimal relay position can be found via solving the following optimization problem

$$
\begin{aligned}
& r_{s l}^{*}=\arg \min _{r_{s l}} \mathcal{P}_{O C}\left(r_{s d}, r_{s l}\right), \\
& \text { subject to } \quad 0 \leq r_{s l} \leq r_{s d} .
\end{aligned}
$$

\footnotetext{
${ }^{2}$ In the above, we used $\mathcal{P}_{O C}$ to denote outage as the calculations are valid for the cooperation protocol with any relay-assignment algorithm.
}

The above optimization problem can be simply analytically solved, and the optimal relay position can be shown to be equal to $r_{s l}^{*}=\frac{r_{s d}}{2}$ for $\eta>1$.

Based on this finding, the Genie-Aided protocol works as follows. For any source node in the network, a Genie is going to put a relay at the mid point of the line joining this source node and the destination (BS/AP). Now, we analyze the average outage performance of the Genie-Aided protocol. We will denote the average probability of outage of this protocol by $\mathcal{P}_{O G}$. Substituting the optimal relay position $r_{s l}^{*}$ in the conditional outage expression (13), and averaging over all possible users' locations,

$$
\mathcal{P}_{O G}=\int_{0}^{\rho} q\left(r_{s d}\right) \mathcal{P}_{O G}\left(r_{s d}\right) d r_{s d},
$$

where $q\left(r_{s d}\right)$ is the distribution of the user location defined in (1), and using the definition of the incomplete gamma function in (8), the average outage probability of the Genie-Aided protocol can be determined as follows

$$
\begin{aligned}
& \mathcal{P}_{O G}=1+\frac{2}{\eta \rho^{2}} \phi^{\frac{2}{\eta}} \Gamma\left(\frac{2}{\eta}, \frac{\rho^{\eta}}{\phi}\right)-\frac{2}{\eta \rho^{2}}\left(\frac{k P_{T C}}{N_{o} \gamma}\right)^{\frac{2}{\eta}} \\
& \times\left[\Gamma\left(\frac{2}{\eta}, \frac{N_{o} \gamma \rho^{\eta}}{k P_{T C}}\right)-\left(2^{1-\eta}\right)^{-\frac{2}{\eta}} \cdot \Gamma\left(\frac{2}{\eta}, \frac{N_{o} \gamma 2^{1-\eta} \rho^{\eta}}{k P_{T C}}\right)\right] .
\end{aligned}
$$

where $\phi=\frac{k P_{T C}}{N_{o} \gamma\left(1+2^{1-\eta}\right)}$. The above results for the GenieAided protocol can be summarized in the following theorem.

Theorem 1: The outage probability $P_{O C}$ of a cooperative transmission scheme utilizing incremental relaying and any practical relay-assignment algorithm, whether centralized or distributed, can be lower bounded as follows

$$
\mathcal{P}_{O G} \leq P_{O C},
$$

where $\mathcal{P}_{O G}$ is given by (16), and the lower bound is achieved by the Genie-Aided protocol.

\section{The BS/AP-Controlled Protocol}

The Genie-Aided protocol is not realizable in a practical network setting, since we cannot have a relay attached to each user during his movement such that the relay is always at the midpoint of the line joining the user and the BS/AP. Nevertheless, the Genie-Aided protocol can lead us to think of a practical relay-assignment protocol that can do the best to achieve the performance lower bound. The knowledge assumed in our proposed protocol is that the BS/AP knows the locations of all of the users attached to its cell. This can be done via positioning and localization algorithms in wireless networks (a survey on these algorithms can be found in [8]). The BS/AP-controlled protocol that we propose works as follows. The BS/AP is going to assign to each user the node that is closest to the mid-point of the line between the user and the BS/AP.

Now, we analyze the performance of the BS/AP-controlled protocol. Assume a source-destination pair that are $r_{s d}$ units 
distance apart as shown in Fig. 2. Let the closet node to the midpoint $c$ between the source and the destination be at a distance $r_{c}$ from $c$. Therefore, the relay $l$ which is assigned to the source lies on a circle of radius $r_{c}$ with center $c$, and the angle that the relay makes with the straight line $s-d$ is $\theta$ as shown in Fig. 2. For this fixed realization of the source, destination, and relay positions, the conditional outage probability can be given in a similar way to (13). From the geometry of the relay position in Fig. 2, the source-relay distance $r_{s l}$ and the relay-destination distance $r_{l d}$ are given by

$$
\begin{aligned}
& r_{s l}=\sqrt{\frac{r_{s d}^{2}}{4}+r_{c}^{2}+r_{s d} r_{c} \cos (\theta)}, \\
& r_{l d}=\sqrt{\frac{r_{s d}^{2}}{4}+r_{c}^{2}-r_{s d} r_{c} \cos (\theta)} .
\end{aligned}
$$

Next, let us find the probability density function of the relay position. Let $x$ be a random variable that denotes the distance of the closet node to point $c$. Then the complementary cumulative distribution function (CCDF) of $x P\left(x \geq r_{c}\right)$, is equal to the probability that the circle of radius $r_{c}$ does not contain any other nodes. Since there are $N$ nodes that are uniformly distributed in a circular cell of radius $\rho$, the $\mathrm{CCDF}$ of $x$ is given by

$$
\mathcal{P}\left(x \geq r_{c}\right)=\left(1-\frac{r_{c}^{2}}{\rho^{2}}\right)^{N},
$$

which is the probability that all of the $N$ users lie outside of the circle. The probability density function of $x$ is thus given by

$\mathcal{P}_{x}\left(r_{c}\right)=\frac{\partial}{\partial r_{c}}\left(1-\left(1-\frac{r_{c}^{2}}{\rho^{2}}\right)^{N}\right)=\frac{2 N r_{c}}{\rho^{2}}\left(1-\frac{r_{c}^{2}}{\rho^{2}}\right)^{N-1}$.

The angle $\theta$ is uniformly distributed between $[0,2 \pi)$. The exact outage probability expression can be found by averaging the conditional outage probability (13) over the user and relay positions given by (1) and (20). The expression, however, is very complicated, so next we derive an upper bound instead.

To derive the upper bound on the BS/AP-controlled protocol, we try to find the worst relay position on a circle of radius $r_{c}$, and then find the outage expression based on that. From Fig. 2, it is obvious that the four quadrants of the circle are symmetric in terms of performance, hence, we will focus on the first quadrant, i.e., the $\operatorname{arc} A B$. We want to find the angle $\theta, 0 \leq \theta \leq \pi / 2$, that maximizes the outage probability expression in (13). From (13) and (18), the outage expression depends on the angle $\theta$ via the quantity

$$
f(\theta)=\left(r_{s l}\right)^{\eta / 2}+\left(r_{l d}\right)^{\eta / 2} .
$$

Maximizing $f(\theta)$ will maximize the outage expression. We can show that for propagation constant $\eta \geq 2$, the function has its global maximum at $\theta=0$. Hence, the worst case relay position is at point $B$, and this should give an upper bound on the outage performance of the BS/AP-controlled protocol. At point $B$, the distances $r_{s l}$ and $r_{l d}$ are given by, $r_{s l}=r_{s d} / 2+r_{c}$ and $r_{l d}=$ $r_{s d} / 2-r_{c}$. An upper bound on the outage probability $\mathcal{P}_{U B}$ of the BS/AP-controlled protocol can thus be found via

$$
\begin{gathered}
\mathcal{P}_{U B} \simeq \int_{0}^{\rho} \int_{0}^{\frac{r_{s d}}{2}} \frac{4 N r_{s d} r_{c}}{\rho^{2}}\left(1-\frac{r_{c}^{2}}{\rho^{2}}\right)^{N-1}\left(1-\exp \left(-\frac{N_{o} \gamma r_{s d}^{\eta}}{K P_{T C}}\right)\right) \\
\cdot\left(1-\exp \left(-\frac{N_{o} \gamma\left(\left(r_{s d} / 2+r_{c}\right)^{\eta}+\left(r_{s d} / 2-r_{c}\right)^{\eta}\right)}{K P_{T C}}\right)\right) d r_{c} d r_{s d} .
\end{gathered}
$$

It is interesting to show that for a propagation constant of $\eta=2$, the upper bound in (22) is equal to the exact outage performance of the BS/AP-controlled protocol. We do not include the details here, however, for lack of space.

The performance of the BS/AP-controlled protocol can be summarized in the following theorem:

Theorem 2: The outage performance of the BS/APcontrolled $\left(P_{O B}\right)$ protocol can be bounded as follows

$$
\mathcal{P}_{O G} \leq \mathcal{P}_{O B} \leq \mathcal{P}_{U B}
$$

where the lower bound is determined by (16) and can be achieved by the Genie-Aided protocol, and the upper bound is specified in (22). The upper bound in (23) becomes an equality if the propagation constant $\eta$ equals 2 .

Theorem 2 provides us with lower and upper bounds on the performance of the BS/AP-controlled protocol. Both these bounds will be shown to be close in the simulations presented in Section IV, specially for the cellular system scenario.

\section{Simulations}

For fairness in comparison between the proposed cooperative schemes and the direct transmission scheme, the average transmitter power is kept fixed in both cases and this is done as follows. Since a packet is either transmitted once or twice in the cooperative protocol, the average transmitted power in the cooperative case $E$ (TX Power) falls between $P_{T C}$ and $2 P_{T C}$. We can prove that the average transmitted power is given by

$$
\begin{aligned}
E(\text { TX Power })=P_{T C} & \left(1+\mathcal{P}_{O D}^{s d}\left(P_{T C}\right)\right. \\
& \left.-\mathcal{P}_{O D}^{s d}\left(P_{T C}\right) \mathcal{P}_{O D}^{s l}\left(P_{T C}\right)\right) .
\end{aligned}
$$

where $\mathcal{P}_{O D}^{s d}\left(P_{T C}\right)$ denotes the outage probability of the direct transmission between the source and the destination when the source is using transmitting power $P_{T C}$ in the cooperative mode, and $\mathcal{P}_{O D}^{s l}\left(P_{T C}\right)$ denotes the corresponding probability for the $s-l$ link. The power used in transmitting in the direct scheme $P_{T D}$ should be set equal to the quantity in (24) in order to have the same average transmitted power. One can see that $P_{T D} \geq P_{T C}$ as expected. In our simulations, we set $P_{T D}=P_{T C}\left(1+\mathcal{P}_{O D}^{s d}\left(P_{T C}\right)\right)$ which is in favor of the direct transmission. A remark on the bandwidth efficiency is now in order. Note that for the cooperative scheme we either utilize the same resources as the direct transmission mode or twice these resources with the same probabilities as in the transmitted power case. This means that the relation between the bandwidth efficiency of the direct and cooperative transmissions is 
also governed in the same manner as for the power in (24). For practical outage performance in the range of 0.01 , the loss in the bandwidth efficiency is thus negligible and we do not take it into account in our simulations.

We considered a cellular system, the cell radius was taken between $1 \mathrm{Km}$ and $10 \mathrm{Km}, N_{o}=-100 \mathrm{dBm}$, and $\eta=3$. The number of users in the cell attached to the BS/AP was taken to be $N=100$ and $\gamma=5 d B$. In Fig. 3, we fix the average transmitted power to $100 \mathrm{~mW}(20 \mathrm{dBm})$ and calculate the outage probability with varying cell size. Furthermore, we plot the outage probability curves computed from the theoretical calculations. As depicted in Fig. 3, if we require the outage probability to be at most 0.01 , the cell radius for direct transmission can be at most $2 \mathrm{~km}$. The BS/AP-controlled protocol can increase the cell radius to $5 \mathrm{~km}$, i.e., $250 \%$ increase in cell size. The theoretical curves match our analytical results: The Genie-Aided protocol provides a lower bound on the outage performance of all the other protocols, and the performance of the BS/AP-controlled protocol is bounded between the upper bound expression $P_{U B}$ and the Genie-Aided protocol as Theorem 2 discussed before. Furthermore, we study the gains that can be achieved from the proposed protocols in terms of energy efficiency. So, we fix the cell radius and calculate the outage probability versus changing the average transmitted power. Fig. 4 depicts the results for a cell radius of $5 \mathrm{~km}$. The average transmitted power is changed from $50 \mathrm{~mW}(17 \mathrm{dBm})$ to $500 \mathrm{~mW}(27 \mathrm{dBm})$. If we still require the outage to be at most 0.01 , then it can be seen from Fig. 4 that the direct transmission scheme can no longer achieve this performance for the whole simulated power range. However, the BS/AP-controlled protocol can achieve this performance at an average transmitted power of $20.5 \mathrm{dBm}$.

\section{Conclusions}

In this paper, we proposed a relay-assignment algorithm to implement a bandwidth efficient cooperation protocol for coverage extension. First, we described a hypothetical Genieaided protocol that acts as a lower bound on the outage performance of any relay-assignment protocol. Next, we proposed the BS/AP-controlled protocol which provides an outage performance close to that of the Genie-Aided scheme. We showed that the bandwidth efficiency loss due to cooperation is negligible for practical operation conditions, for an outage probability of 0.01 the loss in the bandwidth efficiency is approximately 0.01 . Under the same average transmitted power, simulation results reveal an increase in the coverage area up to $250 \%$ in cellular system. Our theoretical calculations match the simulation results.

\section{REFERENCES}

[1] J. N. Laneman, D. N. C. Tse, and G. W. Wornell, "Cooperative diversity in wireless networks: efficient protocols and outage behavior," IEEE Trans. Inform. Theory, vol. 50, pp.3062-3080, Dec. 2004.

[2] J. N. Laneman and G. W. Wornell, "Distributed space-time coded protocols for exploiting cooperative diversity in wireless networks," IEEE Trans. Inform. Theory, vol. 49, pp.2415-2525, Oct. 2003.

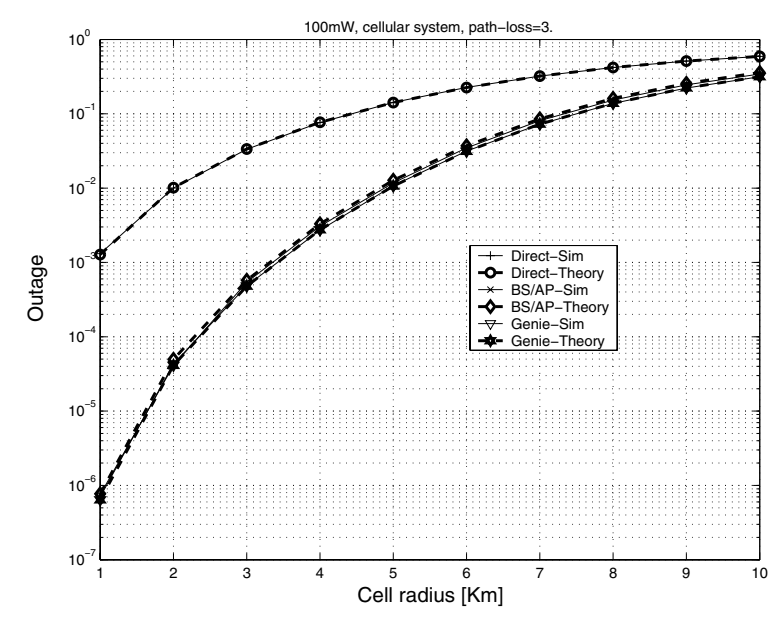

Fig. 3. Average outage probability versus the cell radius in $\mathrm{km}$ for direct and cooperative transmissions. Simulation curves are drawn in solid lines and theoretical curves in dotted lines.

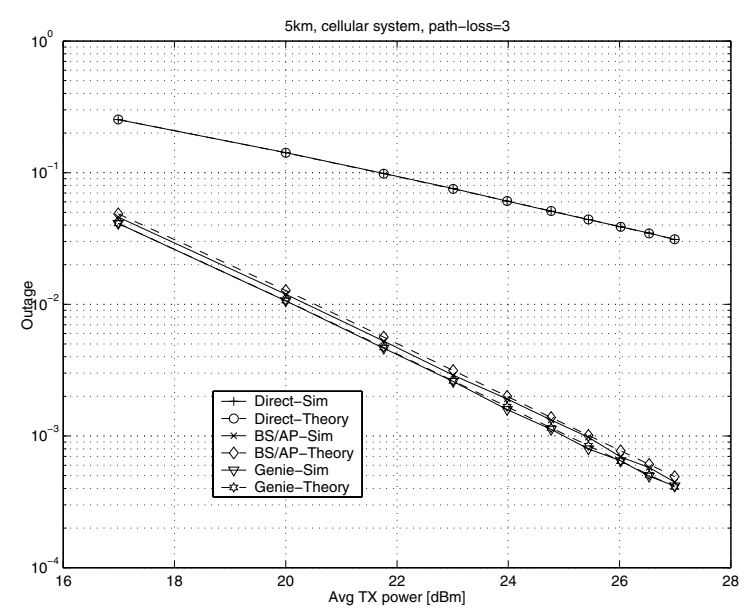

Fig. 4. Average outage probability versus the transmitted power in $d B m$ for direct and cooperative transmissions.

[3] A. Sendonaris, E. Erkip, and B. Aazhang, "User cooperation diversity-Part I: system description," IEEE Trans. Comm., vol. 51, pp.1927-1938, Nov. 2003.

[4] A. Sendonaris, E. Erkip, and B. Aazhang, "User cooperation diversityPart II: implementation aspects and performance analysis," IEEE Trans. Comm., vol. 51, pp.1939-1948, Nov. 2003.

[5] W. Su, A. K. Sadek, and K. J. R. Liu, "SER performance analysis and optimum power allocation for decode-and-forward cooperation protocol in wireless networks," Proc. IEEE Wireless Communications and Networking Conference (WCNC), New Orleans, LA, March 13-17, 2005.

[6] A. K. Sadek, W. Su, and K. J. R. Liu, "A class of cooperative communication protocols for multi-node wireless networks," Proc. IEEE International Workshop on Signal Processing Advances in Wireless Communications (SPAWC), Newyork, June 2005.

[7] J. Boyer, D. D. Falconer, and H. Yanikomeroglu, "Multihop diversity in wireless relaying channels," IEEE Trans. on Communications, vol. 52, no. 10, Oct. 2004.

[8] G. Sun, J. Chen, W. Guo, and K. J. Ray Liu, "Signal processing techniques in network-aided positioning: A survey," IEEE Signal Processing Magazine, vol. 22, no. 4, pp. 12-23, July 2005.

[9] I. S. Gradshteyn and I. M. Ryzhik, "Table of Integrals, Series, and Products," Academic Press, 4-th edition, 1980. 